Supplement of Web Ecol., 20, 19-32, 2020

https://doi.org/10.5194/we-20-19-2020-supplement

(C) Author(s) 2020. This work is distributed under

the Creative Commons Attribution 4.0 License.

(c) (i)

Supplement of

\title{
Pollen morphological variability correlates with a large-scale gradient of aridity
}

\section{Hindel Fatmi et al.}

Correspondence to: Haroun Chenchouni (chenchouni@gmail.com)

The copyright of individual parts of the supplement might differ from the CC BY 4.0 License. 
Table S1. Long-term monthly climatic data of the climatic zones in the region of Tebessa, NE Algeria

\begin{tabular}{|c|c|c|c|c|c|c|c|c|c|c|c|c|c|}
\hline Climate parameters & January & February & March & April & May & June & July & August & September & October & \multicolumn{2}{|c|}{ November December } & Average \\
\hline \multicolumn{14}{|l|}{ Zone 1 (Ms.Med ${ }^{+}$) } \\
\hline Mean temperature $\left[{ }^{\circ} \mathrm{C}\right]$ & $6.7 \pm 0.8$ & $7.7 \pm 0.9$ & $10.1 \pm 1.1$ & $12.9 \pm 1.4$ & $16.8 \pm 1.3$ & $21.2 \pm 1.3$ & $24.7 \pm 1.6$ & $24.8 \pm 1$ & $21.8 \pm 1.1$ & $16.6 \pm 1.1$ & $11.8 \pm 1.1$ & $7.8 \pm 0.9$ & $15.2 \pm 1.1$ \\
\hline Maximum temperature $\left[{ }^{\circ} \mathrm{C}\right]$ & $11.6 \pm 0.8$ & $12.9 \pm 1.6$ & $16.6 \pm 1.1$ & $20.5 \pm 1.3$ & $24.9 \pm 2.4$ & $30.4 \pm 2.7$ & $34.2 \pm 3.1$ & $33.3 \pm 3$ & $29.1 \pm 2.1$ & $22.3 \pm 1.7$ & $17.1 \pm 1.2$ & $12.8 \pm 0.8$ & $22.1 \pm 1.8$ \\
\hline Minimum temperature $\left[{ }^{\circ} \mathrm{C}\right]$ & $2.9 \pm 1.1$ & $3.7 \pm 1.2$ & $5.4 \pm 1.6$ & $7.8 \pm 1.5$ & $12 \pm 1.5$ & $16.3 \pm 1.5$ & $19 \pm 1.3$ & $18.7 \pm 1.1$ & $16.7 \pm 1.1$ & $12.1 \pm 1.6$ & $7.8 \pm 1.8$ & $4.1 \pm 1.8$ & $10.5 \pm 1.4$ \\
\hline Precipitation [mm] & $37.8 \pm 67.3$ & $37.7 \pm 59.5$ & $39.9 \pm 32.1$ & $34.3 \pm 20.8$ & $37.8 \pm 12.3$ & $28.1 \pm 11$ & $17.6 \pm 4.4$ & $16.8 \pm 7.7$ & $22.6 \pm 8.3$ & $33 \pm 18$ & $35.6 \pm 42$ & $36.8 \pm 68.8$ & $31.5 \pm 29.3$ \\
\hline Potential evapotranspiration [mm] & $36.1 \pm 10.7$ & $47 \pm 10.5$ & $76.6 \pm 16$ & $100.6 \pm 20.6$ & $124 \pm 15.2$ & $153.6 \pm 19.4$ & $181.3 \pm 18.1$ & $163.7 \pm 16.1$ & $122 \pm 12$ & $85.1 \pm 12.9$ & $48.4 \pm 9.8$ & $40.1 \pm 11.3$ & $98.2 \pm 14.4$ \\
\hline Ground frost frequency [\%] & 20 & 16 & 9 & 2 & 0 & 0 & 0 & 0 & 0 & 0 & 2 & 14 & 5 \\
\hline Effective rain [mm] & 35 & 35 & 37 & 32 & 36 & 27 & 17 & 16 & 22 & 31 & 34 & 35 & 358 \\
\hline Effective rain ratio [\%] & 94 & 94 & 94 & 95 & 94 & 96 & 97 & 97 & 96 & 95 & 94 & 94 & 95 \\
\hline Rainy days & 7 & 7 & 7 & 6 & 5 & 4 & 2 & 2 & 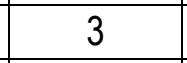 & 5 & 6 & 7 & 61 \\
\hline Solid precipitation ratio [\%] & 8 & 6 & 3 & 1 & 0 & 0 & 0 & 0 & 0 & 0 & 2 & 6 & 3 \\
\hline \multicolumn{14}{|l|}{ Zone 2 (Th.Med-') } \\
\hline Mean temperature $\left[{ }^{\circ} \mathrm{C}\right]$ & $6.7 \pm 0.7$ & $7.6 \pm 0.8$ & $10.7 \pm 1$ & $13.6 \pm 1.5$ & $17.7 \pm 2$ & $23 \pm 2$ & $26.2 \pm 2.7$ & $25.9 \pm 2.1$ & $22.4 \pm 1.9$ & $16 \pm 1.5$ & $11.2 \pm 1$ & $8 \pm 0.8$ & $15.8 \pm 1.5$ \\
\hline Maximum temperature $\left[{ }^{\circ} \mathrm{C}\right]$ & $10.8 \pm 0.7$ & $12.3 \pm 1.3$ & $16.2 \pm 1.1$ & $20.5 \pm 1.3$ & $25 \pm 2.4$ & $31 \pm 2.3$ & $34.9 \pm 3.2$ & $33.8 \pm 3$ & $29.3 \pm 1.9$ & $21.8 \pm 1.6$ & $16.3 \pm 1.1$ & $11.8 \pm 0.9$ & $22 \pm 1.7$ \\
\hline Minimum temperature $\left[{ }^{\circ} \mathrm{C}\right]$ & $1.9 \pm 1.1$ & $2.9 \pm 1.1$ & $4.6 \pm 1.5$ & $7.3 \pm 1.5$ & $11.7 \pm 1.7$ & $16.2 \pm 1.8$ & $18.9 \pm 1.7$ & $18.3 \pm 1.4$ & $16.2 \pm 1.3$ & $11.3 \pm 1.6$ & $6.8 \pm 1.6$ & $3 \pm 1.5$ & $9.9 \pm 1.5$ \\
\hline Precipitation [mm] & $28.7 \pm 69.1$ & $31.7 \pm 56.4$ & $45.8 \pm 38$ & $34.6 \pm 23.8$ & $39.7 \pm 11.7$ & $26.4 \pm 13.2$ & $9.8 \pm 5.4$ & $25.5 \pm 6.3$ & $37.1 \pm 10.6$ & $28.2 \pm 21.2$ & $32.5 \pm 42.9$ & $25.7 \pm 71.6$ & $30.5 \pm 30.8$ \\
\hline Potential evapotranspiration [mm] & $33.2 \pm 11.6$ & $45.2 \pm 11.4$ & $75.3 \pm 23$ & $100.2 \pm 27.7$ & $123 \pm 26.3$ & $153.7 \pm 28.9$ & $181.6 \pm 28.6$ & $165.3 \pm 21.9$ & $122.5 \pm 17.3$ & $83.1 \pm 19.9$ & $45.6 \pm 14.9$ & $38.3 \pm 13.2$ & $97.3 \pm 20.4$ \\
\hline Ground frost frequency [\%] & 25 & 20 & 12 & 3 & 0 & 0 & 0 & 0 & 0 & 0 & 4 & 20 & 7 \\
\hline Effective rain [mm] & 27 & 30 & 42 & 33 & 37 & 25 & 10 & 24 & 35 & 27 & 31 & 25 & 346 \\
\hline Effective rain ratio [\%] & 95 & 95 & 93 & 94 & 94 & 96 & 98 & 96 & 94 & 95 & 95 & 96 & 95 \\
\hline Rainy days & 5 & 6 & 8 & 5 & 6 & 3 & 1 & 3 & 5 & 4 & 5 & 5 & 56 \\
\hline Solid precipitation ratio [\%] & 8 & 6 & 2 & 1 & 0 & 0 & 0 & 0 & 0 & 1 & 2 & 5 & 2 \\
\hline \multicolumn{14}{|l|}{ Zone 3 (Th.Med ${ }^{+}$) } \\
\hline Mean temperature $\left[{ }^{\circ} \mathrm{C}\right]$ & $6.5 \pm 1.1$ & $7.5 \pm 1$ & $10.6 \pm 1.2$ & $13.5 \pm 1.3$ & $17.5 \pm 1.3$ & $22.8 \pm 1.5$ & $26 \pm 1.8$ & $25.7 \pm 1.8$ & $22.2 \pm 1.7$ & $15.8 \pm 1.5$ & $11.1 \pm 1.2$ & $7.9 \pm 1$ & $15.6 \pm 1.4$ \\
\hline Maximum temperature $\left[{ }^{\circ} \mathrm{C}\right]$ & $10.6 \pm 2.5$ & $12.1 \pm 2.4$ & $16.1 \pm 2.5$ & $20.5 \pm 2.6$ & $25 \pm 3$ & $31.1 \pm 3.5$ & $35 \pm 3.7$ & $33.9 \pm 3.4$ & $29.3 \pm 2.4$ & $21.7 \pm 2.5$ & $16.1 \pm 2.3$ & $11.6 \pm 2.5$ & $21.9 \pm 2.8$ \\
\hline Minimum temperature $\left[{ }^{\circ} \mathrm{C}\right]$ & $1.7 \pm 2.5$ & $2.7 \pm 2.3$ & $4.4 \pm 2.7$ & $7.1 \pm 3$ & $11.6 \pm 3.2$ & $16.1 \pm 3.5$ & $18.8 \pm 3.5$ & $18.2 \pm 3.3$ & $16.1 \pm 2.5$ & $11.1 \pm 2.8$ & $6.6 \pm 2.9$ & $2.7 \pm 2.8$ & $9.8 \pm 2.9$ \\
\hline Precipitation [mm] & $27 \pm 21.1$ & $30 \pm 26.8$ & $46 \pm 25.7$ & $34 \pm 15.6$ & $40 \pm 12.7$ & $27 \pm 10.2$ & $10 \pm 3.6$ & $26 \pm 5.7$ & $37 \pm 10.1$ & $28 \pm 8.3$ & $32 \pm 14.4$ & $24 \pm 26.1$ & $30.1 \pm 15$ \\
\hline Potential evapotranspiration [mm] & $32.6 \pm 11.1$ & $44.7 \pm 12.4$ & $74.8 \pm 22.8$ & $99.9 \pm 25.8$ & $122.5 \pm 27.3$ & $153.5 \pm 29.5$ & $181.4 \pm 27$. & $165.4 \pm 22.9$ & $122.4 \pm 18$ & $82.5 \pm 19.7$ & $44.9 \pm 17.4$ & $37.9 \pm 12.3$ & $96.9 \pm 20.6$ \\
\hline
\end{tabular}




\begin{tabular}{|c|c|c|c|c|c|c|c|c|c|c|c|c|c|}
\hline Ground frost frequency [\%] & 27 & 21 & 13 & 4 & 0 & 0 & 0 & 0 & 0 & 0 & 5 & 21 & 7 \\
\hline Effective rain [mm] & 26 & 29 & 43 & 32 & 37 & 26 & 10 & 25 & 35 & 27 & 30 & 23 & 342 \\
\hline Effective rain ratio [\%] & 96 & 95 & 93 & 95 & 94 & 96 & 98 & 96 & 94 & 96 & 95 & 96 & 95 \\
\hline Rainy days & 5 & 5 & 8 & 5 & 6 & 3 & 1 & 3 & 5 & 4 & 5 & 4 & 54 \\
\hline Solid precipitation ratio [\%] & 8 & 6 & 3 & 1 & 0 & 0 & 0 & 0 & 0 & 1 & 2 & 5 & 2 \\
\hline \multicolumn{14}{|l|}{ Zone 4 (X.T.Med) } \\
\hline Mean temperature $\left[{ }^{\circ} \mathrm{C}\right]$ & $5.7 \pm 0.8$ & $6.6 \pm 0.8$ & $9.6 \pm 0.9$ & $12.4 \pm 1.5$ & $16.3 \pm 1.5$ & $21.7 \pm 2$ & $24.8 \pm 2.2$ & $24.5 \pm 1.8$ & $21.1 \pm 1.5$ & $14.8 \pm 1.5$ & $10.3 \pm 1$ & $7.1 \pm 0.6$ & $14.6 \pm 1.4$ \\
\hline Maximum temperature $\left[{ }^{\circ} \mathrm{C}\right]$ & $9.7 \pm 0.7$ & $11.2 \pm 1.1$ & $15.4 \pm 1.5$ & $20 \pm 2.1$ & $24.5 \pm 2.5$ & $31.1 \pm 3.6$ & $35 \pm 3.8$ & $33.8 \pm 3.3$ & $29.1 \pm 2.4$ & $21 \pm 1.6$ & $15.2 \pm 1.2$ & $10.7 \pm 1$ & $21.4 \pm 2.1$ \\
\hline Minimum temperature $\left[{ }^{\circ} \mathrm{C}\right]$ & $0.8 \pm 0.8$ & $1.9 \pm 1$ & $3.6 \pm 1.6$ & $6.3 \pm 2.2$ & $10.9 \pm 2.7$ & $15.6 \pm 3.2$ & $18.3 \pm 3.1$ & $17.5 \pm 2.6$ & $15.5 \pm 1.9$ & $10.3 \pm 1.6$ & $5.7 \pm 1.5$ & $1.7 \pm 1.5$ & $9 \pm 2$ \\
\hline Precipitation [mm] & $31.3 \pm 13.7$ & $34.6 \pm 10.6$ & $52.6 \pm 14.5$ & $37.9 \pm 7.7$ & $45.6 \pm 8$ & $30.1 \pm 13.7$ & $11.7 \pm 4.5$ & $28 \pm 7.1$ & $39.8 \pm 7.5$ & $31.9 \pm 4.8$ & $37.2 \pm 7.3$ & $28.7 \pm 12.2$ & $34.1 \pm 9.3$ \\
\hline Potential evapotranspiration [mm] & $29.3 \pm 11.6$ & $41.6 \pm 12.9$ & $71.3 \pm 23.6$ & $96.7 \pm 27.4$ & $117.3 \pm 28.2$ & $150.8 \pm 30.6$ & $178 \pm 29.9$ & $163.9 \pm 24.4$ & $120.6 \pm 19.1$ & $78.4 \pm 20.4$ & $40.5 \pm 16.8$ & $36.4 \pm 13.5$ & $93.7 \pm 21.5$ \\
\hline Ground frost frequency [\%] & 32 & 26 & 17 & 6 & 0 & 0 & 0 & 0 & 0 & 0 & 8 & 27 & 9 \\
\hline Effective rain [mm] & 30 & 33 & 48 & 36 & 42 & 29 & 11 & 27 & 37 & 30 & 35 & 27 & 385 \\
\hline Effective rain ratio [\%] & 95 & 94 & 92 & 94 & 93 & 95 & 98 & 96 & 94 & 95 & 94 & 95 & 94 \\
\hline Rainy days & 6 & 6 & 9 & 6 & 7 & 4 & 1 & 3 & 5 & 5 & 6 & 5 & 63 \\
\hline Solid precipitation ratio [\%] & 10 & 8 & 3 & 1 & 0 & 0 & 0 & 0 & 0 & 1 & 3 & 7 & 3 \\
\hline \multicolumn{14}{|l|}{ Zone 5 (Sb.Des') } \\
\hline Mean temperature $\left[{ }^{\circ} \mathrm{C}\right]$ & $9.8 \pm 1.1$ & $11.6 \pm 1$ & $15.1 \pm 1.5$ & $19 \pm 2$ & $23.3 \pm 1.8$ & $27.8 \pm 2.4$ & $31 \pm 2.6$ & $30.7 \pm 2.3$ & $27.1 \pm 2.1$ & $21.8 \pm 1.8$ & $15.6 \pm 1.2$ & $10.8 \pm 0.8$ & $20.3 \pm 1.7$ \\
\hline Maximum temperature $\left[{ }^{\circ} \mathrm{C}\right]$ & $15.8 \pm 2.4$ & $19 \pm 2.4$ & $22 \pm 2.4$ & $25.7 \pm 2.8$ & $31.1 \pm 3.3$ & $34.7 \pm 3.5$ & $39 \pm 3.9$ & $38.2 \pm 3.4$ & $32.7 \pm 2$ & $27.6 \pm 2.4$ & $21.2 \pm 2.2$ & $15.6 \pm 2.3$ & $26.9 \pm 2.8$ \\
\hline Minimum temperature $\left[{ }^{\circ} \mathrm{C}\right]$ & $4.5 \pm 2.3$ & $6.8 \pm 2.1$ & $9.3 \pm 2.6$ & $12.6 \pm 3.1$ & $17.6 \pm 3.3$ & $21.7 \pm 3.9$ & $24.2 \pm 3.6$ & $24.6 \pm 3.5$ & $20.7 \pm 2.9$ & $15.6 \pm 2.7$ & $10 \pm 2.3$ & $5 \pm 2.3$ & $14.4 \pm 2.9$ \\
\hline Precipitation [mm] & $15 \pm 13.2$ & $11 \pm 13.2$ & $16 \pm 17.1$ & $14 \pm 9.4$ & $11 \pm 12.2$ & $5 \pm 11.7$ & $1 \pm 3.5$ & $7 \pm 5.7$ & $14 \pm 10.9$ & $13 \pm 8.3$ & $17 \pm 8.9$ & $13 \pm 14.2$ & $11.4 \pm 10.7$ \\
\hline Potential evapotranspiration [mm] & $53.8 \pm 8.7$ & $64.9 \pm 11.1$ & $116.3 \pm 20.8$ & $145.1 \pm 24.4$ & $191.6 \pm 29.2$ & $205.8 \pm 28.7$ & $237.6 \pm 32.2$ & $212.3 \pm 22.7$ & $159.8 \pm 17.7$ & $123.1 \pm 19.8$ & $80 \pm 14.7$ & $54.9 \pm 21.4$ & $137.1 \pm 20.9$ \\
\hline Ground frost frequency [\%] & 12 & 4 & 0 & 0 & 0 & 0 & 0 & 0 & 0 & 0 & 0 & 10 & 2 \\
\hline Effective rain [mm] & 15 & 11 & 16 & 14 & 11 & 5 & 1 & 7 & 14 & 13 & 17 & 13 & 134 \\
\hline Effective rain ratio [\%] & 98 & 98 & 97 & 98 & 98 & 99 & 100 & 99 & 98 & 98 & 97 & 98 & 98 \\
\hline Rainy days & 3 & 2 & 3 & 2 & 1 & 1 & 0 & 1 & 2 & 2 & 3 & 2 & 22 \\
\hline Solid precipitation ratio [\%] & 3 & 2 & 1 & 0 & 0 & 0 & 0 & 0 & 0 & 0 & 1 & 2 & 1 \\
\hline \multicolumn{14}{|l|}{ Zone 6 (Sb.Des') } \\
\hline Mean temperature $\left[{ }^{\circ} \mathrm{C}\right]$ & $9.8 \pm 1.3$ & $11.6 \pm 1.3$ & $15.1 \pm 1.4$ & $19 \pm 2.2$ & $23.3 \pm 2.1$ & $27.8 \pm 3$ & $31 \pm 3$ & $30.7 \pm 2.7$ & $27.1 \pm 2.2$ & $21.8 \pm 1.9$ & $15.6 \pm 1.3$ & $10.8 \pm 0.9$ & $20.3 \pm 1.9$ \\
\hline Maximum temperature $\left[{ }^{\circ} \mathrm{C}\right]$ & $15.8 \pm 2.6$ & $19 \pm 2.5$ & $22 \pm 2.5$ & $25.7 \pm 2.7$ & $31.1 \pm 3.3$ & $34.7 \pm 3.4$ & $39 \pm 4.2$ & $38.2 \pm 3.7$ & $32.7 \pm 2.3$ & $27.6 \pm 2.5$ & $21.2 \pm 2.3$ & $15.6 \pm 2.7$ & $26.9 \pm 2.9$ \\
\hline Minimum temperature $\left[{ }^{\circ} \mathrm{C}\right]$ & $4.5 \pm 2.7$ & $6.8 \pm 2.5$ & $9.3 \pm 3.1$ & $12.6 \pm 3.6$ & $17.6 \pm 3.6$ & $21.7 \pm 4.1$ & $24.2 \pm 3.8$ & $24.6 \pm 3.7$ & $20.7 \pm 3.2$ & $15.6 \pm 3.2$ & $10 \pm 3$ & $5 \pm 2.8$ & $14.4 \pm 3.3$ \\
\hline Precipitation [mm] & $15 \pm 16.9$ & $11 \pm 14.8$ & $16 \pm 24$ & $14 \pm 13$ & $11 \pm 13.4$ & $5 \pm 12.7$ & $1 \pm 3.5$ & $7 \pm 5.7$ & $14 \pm 9.4$ & $13 \pm 8.1$ & $17 \pm 12.2$ & $13 \pm 14.7$ & $11.4 \pm 12.4$ \\
\hline
\end{tabular}




\begin{tabular}{|c|c|c|c|c|c|c|c|c|c|c|c|c|c|}
\hline Potential evapotranspiration [mm] & $53.8 \pm 9.4$ & $64.9 \pm 10.4$ & $116.3 \pm 21.7$ & $145.1 \pm 24.4$ & $191.6 \pm 28.1$ & $205.8 \pm 27.1$ & $237.6 \pm 30.7$ & $212.3 \pm 23.3$ & $159.8 \pm 17$ & $123.1 \pm 19.4$ & $80 \pm 15.3$ & $54.9 \pm 19$ & $137.1 \pm 20.5$ \\
\hline Ground frost frequency [\%] & 12 & 4 & 0 & 0 & 0 & 0 & 0 & 0 & 0 & 0 & 0 & 10 & 2 \\
\hline Effective rain [mm] & 15 & 11 & 16 & 14 & 11 & 5 & 1 & 7 & 14 & 13 & 17 & 13 & 134 \\
\hline Effective rain ratio [\%] & 98 & 98 & 97 & 98 & 98 & 99 & 100 & 99 & 98 & 98 & 97 & 98 & 98 \\
\hline Rainy days & 3 & 2 & 3 & 2 & 1 & 1 & 0 & 1 & 2 & 2 & 3 & 2 & 22 \\
\hline Solid precipitation ratio [\%] & 3 & 2 & 1 & 0 & 0 & 0 & 0 & 0 & 0 & 0 & 1 & 2 & 1 \\
\hline \multicolumn{14}{|l|}{ Zone 7 (Desert) } \\
\hline Mean temperature $\left[{ }^{\circ} \mathrm{C}\right]$ & $10.1 \pm 0.9$ & $11.9 \pm 0.9$ & $15.4 \pm 1.2$ & $19.6 \pm 1.8$ & $23.7 \pm 1.6$ & $28.4 \pm 2.6$ & $31.6 \pm 2.6$ & $31.4 \pm 2.2$ & $27.9 \pm 1.8$ & $21.8 \pm 1.4$ & $15.8 \pm 0.9$ & $11.2 \pm 0.9$ & $20.7 \pm 1.6$ \\
\hline Maximum temperature $\left[{ }^{\circ} \mathrm{C}\right]$ & $15.3 \pm 0.5$ & $18.5 \pm 0.6$ & $21.6 \pm 1.5$ & $25.3 \pm 2.1$ & $30.7 \pm 2.4$ & $34.4 \pm 2.6$ & $38.7 \pm 3.2$ & $37.9 \pm 2.6$ & $32.4 \pm 1.2$ & $27.1 \pm 0.5$ & $20.7 \pm 0.5$ & $15.2 \pm 0.8$ & $26.5 \pm 1.5$ \\
\hline Minimum temperature $\left[{ }^{\circ} \mathrm{C}\right]$ & $4.2 \pm 1.5$ & $6.4 \pm 1.4$ & $8.9 \pm 2.1$ & $12.2 \pm 2.8$ & $17.2 \pm 3.2$ & $21.3 \pm 3.8$ & $23.8 \pm 3.4$ & $24.2 \pm 3$ & $20.3 \pm 2.7$ & $15.2 \pm 2.1$ & $9.6 \pm 1.7$ & $4.7 \pm 1.4$ & $14 \pm 2.4$ \\
\hline Precipitation [mm] & $11.9 \pm 14.6$ & $10.1 \pm 13$ & $16.1 \pm 22.3$ & $11.6 \pm 11.9$ & $7.6 \pm 11.5$ & $4.4 \pm 11.3$ & $0.8 \pm 4.3$ & $3 \pm 6.7$ & $8.3 \pm 6.6$ & $10.8 \pm 7.1$ & $14.3 \pm 11.3$ & $12 \pm 9.9$ & $9.2 \pm 10.9$ \\
\hline Potential evapotranspiration [mm] & $52.1 \pm 14$ & $63.4 \pm 15.6$ & $114.4 \pm 27.2$ & $143.6 \pm 31.1$ & $189.4 \pm 33.1$ & $204.7 \pm 31.9$ & $236.5 \pm 36$ & $211.7 \pm 25.5$ & $158.9 \pm 20.9$ & $121.4 \pm 23.5$ & $78.1 \pm 17.9$ & $54 \pm 20.5$ & $135.7 \pm 24.8$ \\
\hline Ground frost frequency [\%] & 14 & 5 & 1 & 0 & 0 & 0 & 0 & 0 & 0 & 0 & 0 & 12 & 3 \\
\hline Effective rain [mm] & 12 & 10 & 16 & 11 & 7 & 4 & 1 & 3 & 8 & 11 & 14 & 12 & 109 \\
\hline Effective rain ratio [\%] & 98 & 98 & 97 & 98 & 99 & 99 & 100 & 100 & 99 & 98 & 98 & 98 & 98 \\
\hline Rainy days & 2 & 2 & 3 & 2 & 1 & 0 & 0 & 0 & 1 & 1 & 2 & 2 & 16 \\
\hline Solid precipitation ratio [\%] & 3 & 2 & 1 & 0 & 0 & 0 & 0 & 0 & 0 & 0 & 1 & 2 & 1 \\
\hline
\end{tabular}

